\title{
Antiproliferative, Ultrastructural, and Physiological Effects of Amiodarone on Promastigote and Amastigote Forms of Leishmania amazonensis
}

\author{
Sara Teixeira de Macedo-Silva,, ${ }^{1,2}$ Thais Larissa Araújo de Oliveira Silva,, ${ }^{1,2}$ \\ Julio A. Urbina, ${ }^{3}$ Wanderley de Souza, ${ }^{1,2,4}$ and Juliany Cola Fernandes Rodrigues ${ }^{1,2,5}$ \\ ${ }^{1}$ Laboratório de Ultraestrutura Celular Hertha Meyer, Instituto de Biofísica Carlos Chagas Filho, \\ Universidade Federal do Rio de Janeiro, Avenida Carlos Chagas, 373, CCS, Ilha do Fundão, 21941-902 Rio de Janeiro, Brazil \\ ${ }^{2}$ Instituto Nacional de Ciência e Tecnologia de Biologia Estrutural e Bioimagem, Brazil \\ ${ }^{3}$ Instituto Venezolano de Investigaciones Cientificas, Caracas, Venezuela \\ ${ }^{4}$ Instituto Nacional de Metrologia, Normalização e Qualidade Industrial (Inmetro), 20261-232 Rio de Janeiro, Brazil \\ ${ }^{5}$ Pólo Avançado de Xerém, Universidade Federal do Rio de Janeiro, 25250-470 Rio de Janeiro, Brazil
}

Correspondence should be addressed to Juliany Cola Fernandes Rodrigues, julycola@biof.ufrj.br

Received 18 January 2011; Revised 1 March 2011; Accepted 14 March 2011

Academic Editor: Kwang Poo Chang

Copyright (c) 2011 Sara Teixeira de Macedo-Silva et al. This is an open access article distributed under the Creative Commons Attribution License, which permits unrestricted use, distribution, and reproduction in any medium, provided the original work is properly cited.

\begin{abstract}
Amiodarone (AMIO), the most frequently antiarrhythmic drug used for the symptomatic treatment of chronic Chagas' disease patients with cardiac compromise, has recently been shown to have also specific activity against fungi, Trypanosoma cruzi and Leishmania. In this work, we characterized the effects of AMIO on proliferation, mitochondrial physiology, and ultrastructure of Leishmania amazonensis promastigotes and intracellular amastigotes. The $\mathrm{IC}_{50}$ values were 4.21 and $0.46 \mu \mathrm{M}$ against promastigotes and intracellular amastigotes, respectively, indicating high selectivity for the clinically relevant stage. We also found that treatment with AMIO leads to a collapse of the mitochondrial membrane potential $(\Delta \Psi m)$ and to an increase in the production of reactive oxygen species, in a dose-dependent manner. Fluorescence microscopy of cells labeled with JC-1, a marker for mitochondrial energization, and transmission electron microscopy confirmed severe alterations of the mitochondrion, including intense swelling and modification of its membranes. Other ultrastructural alterations included (1) presence of numerous lipid-storage bodies, (2) presence of large autophagosomes containing part of the cytoplasm and membrane profiles, sometimes in close association with the mitochondrion and endoplasmic reticulum, and (3) alterations in the chromatin condensation and plasma membrane integrity. Taken together, our results indicate that AMIO is a potent inhibitor of L. amazonensis growth, acting through irreversible alterations in the mitochondrial structure and function, which lead to cell death by necrosis, apoptosis and/or autophagy.
\end{abstract}

\section{Introduction}

Leishmaniasis is a parasitosis caused by different species of the Leishmania genus that affects about 12 million people around the world, with $90 \%$ of the cases reported in Afghanistan, Pakistan, Iran, Iraq, Syria, Saudi Arabia, India, Bangladesh, Nepal, Sudan, Algeria, Ethiopia, Brazil, Bolivia, Colombia, Ecuador, Peru, and Venezuela [1]. Approximately 21 species have been described to cause three different clinical manifestations: (1) cutaneous (CL), where the lesions are confined to the site of the inoculation by the sandfly; (2) mucocutaneous (MCL), which affects the mucosal tissues; (3) visceral (VL), where the parasites have a tropism for phagocytes mainly localized in the spleen and the liver. Visceral leishmaniasis is fatal if not treated, while some forms of cutaneous manifestations can cure spontaneously. According to WHO, around 70,000 deaths per year occur throughout the world [1]. In Brazil, Leishmania amazonensis is one of the species responsible for the cutaneous disease; however, in some individuals the immune system fails to 
mount an appropriate response against the parasite, leading to clinical manifestations of diffuse cutaneous leishmaniasis [2].

Pentavalent antimonials such as meglumine antimoniate (Glucantime) and sodium stibogluconate (Pentostan) have been employed as first-line treatment for many decades [3]. In the case of resistance to pentavalent antimonials, secondline treatments using amphotericin B or pentamidine have been successfully used [4]. For visceral leishmaniasis, miltefosine (Impavido) has been recently employed by oral route in India as a first-line treatment [5]. However, the drug is teratogenic and has a narrow chemotherapeutic window [6]. More recently, combination treatments are emerging as first-line treatments for visceral leishmaniasis [7]. Toxic side effects and increasing resistance limit most of the current specific treatments for leishmaniasis, indicating that there is an urgent need to develop new drugs that are efficacious, safe, and more accessible for the patient populations.

Amiodarone (AMIO) is the antiarrhythmic class III drug most frequently used to treat arrhythmias in general as well as in patients with chronic Chagas' disease and cardiac compromise. The antiarrhythmic action in mammals has been well characterized and results from $\mathrm{Ca}^{2+}$ and $\mathrm{K}^{+}$channel inactivation, but it has recently been shown that the drug also has selective activity against parasitic protozoa such as Trypanosoma cruzi and Leishmania mexicana [8-10] as well as a broad-spectrum antifungal action [11, 12]. The mechanisms of action of AMIO reported in these different microorganisms involve the inhibition of sterol biosynthesis, disruption of mitochondrial membrane potential $(\Delta \Psi m)$, and $\mathrm{Ca}^{2+}$ homeostasis, as well as production of reactive oxygen species $[8,9,11-13]$. Apparently, these alterations in the mitochondrial metabolism trigger a sequence of cellular events leading to apoptosis-like cell death [13]. However, the action of AMIO on the mitochondrion of target cells is controversial, as some groups have implicated inhibition of mitochondrial respiration, mainly by the direct action of AMIO against complex I, II, and F0F1-ATP synthase [13] or by a rapid release of $\mathrm{Ca}^{2+}$ from the mitochondrial compartment due to a collapse of mitochondrial membrane potential $(\Delta \Psi m)$ (see $[8,9])$, while others suggest that AMIO is able to protect mitochondrial function [14-17].

In this study, we investigated the antiproliferative, ultrastructural, and physiological effects of amiodarone on promastigote and intracellular amastigote forms of Leishmania amazonensis. The results indicated that AMIO acts mainly by altering the mitochondrial ultrastructure and physiology but other deleterious effects were also observed, including lipid accumulation, loss of the plasma membrane integrity, and presence of autophagic-like structures, suggesting different types of cell death involved in the mechanism of action of AMIO.

\section{Material and Methods}

2.1. Parasites. MHOM/BR/75/Josefa strain of Leishmania amazonensis isolated from a patient with diffuse cutaneous leishmaniasis by C. A. Cuba-Cuba (Universidade de Brasilia, Brazil) was used in the present study. It has been maintained by hamster footpad inoculation and, in the case of promastigotes, axenically cultured in Warren's medium (brain heart infusion plus hemin and folic acid) [18] supplemented with $10 \%$ fetal bovine serum at $25^{\circ} \mathrm{C}$. Infective promastigotes of the Josefa strain were used to obtain intracellular amastigotes in macrophage cultures.

2.2. Drug. Amiodarone (AMIO), \{(2-butyl-3-benzofuranyl) -[4-[2-(diethylamino)ethoxi]-3,5-diiodophenyl]methanone hydrochloride\}, was purchased from Sigma, dissolved in dimethyl sulfoxide as a $100 \mathrm{mM}$ stock, and stored at $-20^{\circ} \mathrm{C}$.

2.3. In Vitro Antiproliferative Activities of Amiodarone. Growth experiments with promastigotes were initiated with $2.0 \times 10^{6}$ parasites $/ \mathrm{mL}$, and AMIO was added at different concentrations from concentrated stock solutions in DMSO after $24 \mathrm{~h}$ of growth. Cell densities were evaluated daily in a Neubauer chamber during $72 \mathrm{~h}$ of growth. To evaluate the effects of the AMIO on the L. amazonensis intracellular amastigotes, peritoneal macrophages from CF1 mice were harvested by washing them with RPMI medium (Gibco) and plated in 24-well tissue culture chamber slides, allowing them to adhere to the slides for $24 \mathrm{~h}$ at $37^{\circ} \mathrm{C}$ in $5 \% \mathrm{CO}_{2}$. Adherent macrophages were infected with metacyclic promastigotes at a macrophage-to-parasite ratio of $1: 10$ at $35^{\circ} \mathrm{C}$ for $2 \mathrm{~h}$. After this time, noningested parasites were removed by washing and infected cultures were incubated for $24 \mathrm{~h}$ in RPMI (containing 10\% of fetal bovine serum) without AMIO. Different concentrations of AMIO were added after $24 \mathrm{~h}$ of interaction, when the number of amastigotes per macrophage was in the range of two to four, and fresh medium with AMIO was added daily for 2 days. The cultures were fixed with $4 \%$ freshly prepared formaldehyde in phosphate buffer saline (PBS, $\mathrm{pH}$ 7.2) and stained with Giemsa for $15 \mathrm{~min}$. The percentage of infected cells was determined by light microscopy. Association indexes (mean number of parasites internalized per cell, multiplied by the percentage of infected macrophages, and divided by the total number of macrophages) were determined and used as a parameter to calculate the intensity of infection in each condition used in this study. The $50 \%$ inhibitory concentrations $\left(\mathrm{IC}_{50} \mathrm{~s}\right)$ were calculated with the SigmaPlot (version 10) program. The results are expressed as the means of three independent experiments.

2.4. Tests of Viability in Macrophages. To evaluate the cytotoxicity effects of AMIO against the host cell cultures, macrophages were incubated with different concentrations of AMIO for $48 \mathrm{~h}$, and exclusion tests with $0.1 \%$ trypan blue were carried out for $5 \mathrm{~min}$. The percentages of dead and alive cells were determined after counting of 400 macrophages in randomly selected fields by light microscopy.

2.5. Estimation of Mitochondrial Transmembrane Electric Potential $(\Delta \Psi m) . \Delta \Psi m$ of the control and AMIO-treated $(6,10$, and $15 \mu \mathrm{M})$ promastigotes was investigated using 

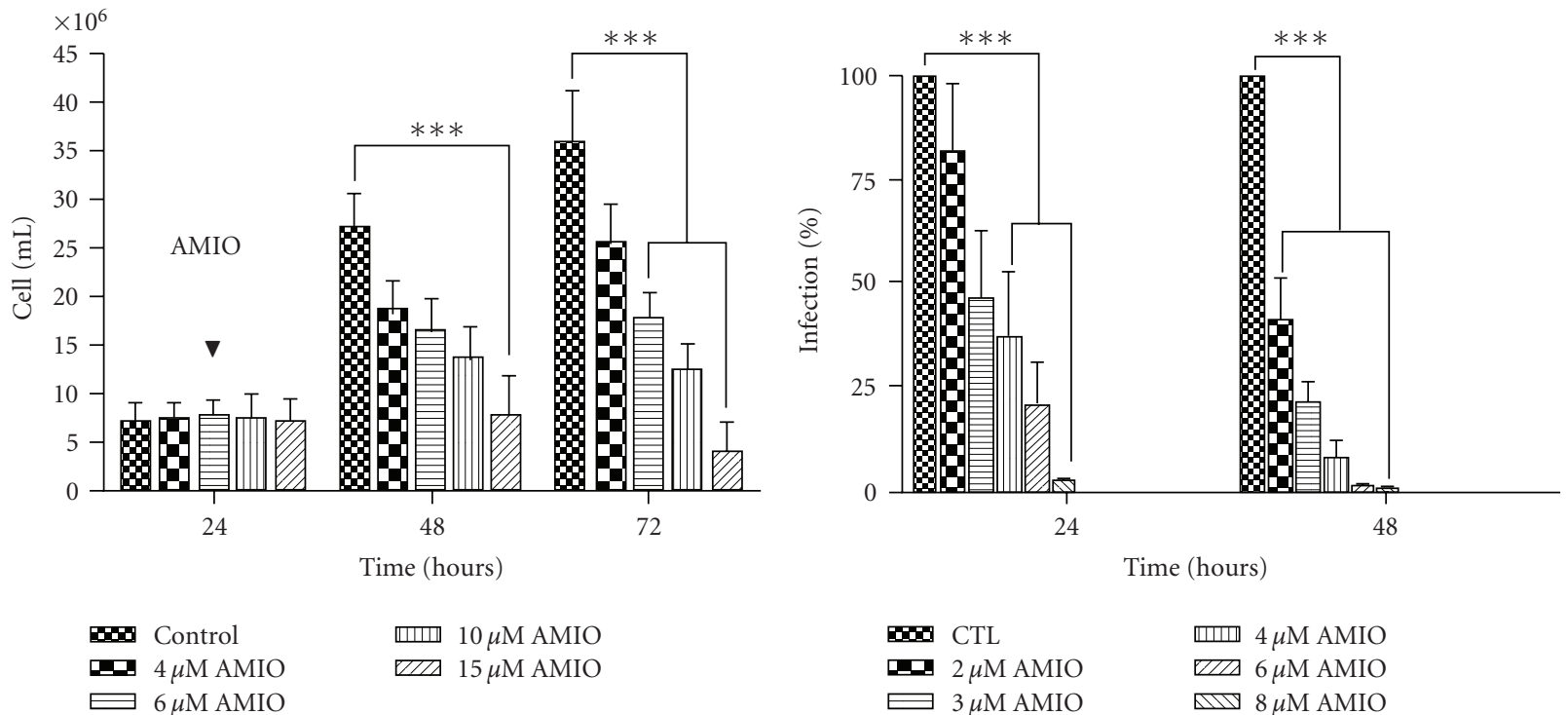

(a)
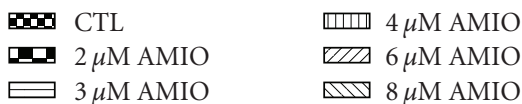

MIV $8 \mu \mathrm{M}$ AMIO

(b)

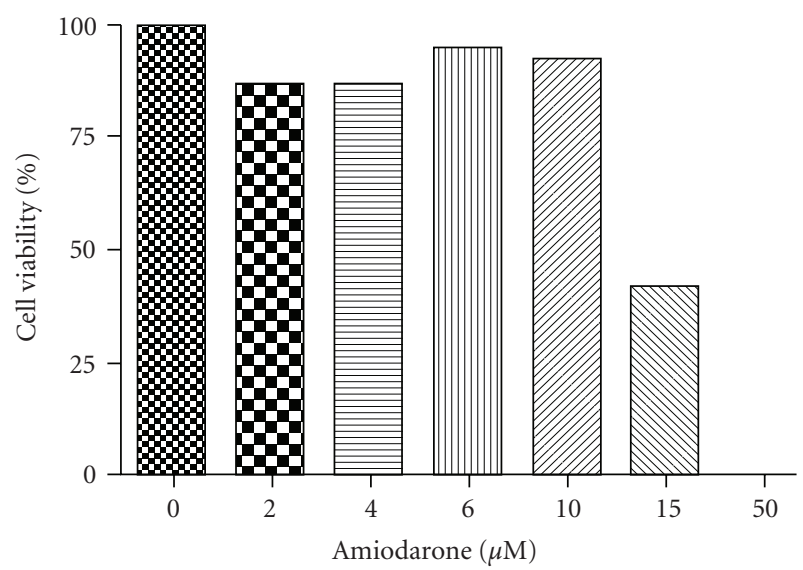

(c)

FIGURE 1: Antiproliferative and cytotoxic effects of amiodarone (AMIO) on Leishmania amazonensis promastigotes and intracellular amastigotes. (a) Treatment of promastigotes with different concentrations of AMIO for $48 \mathrm{~h}$. (b) Effects of amiodarone on intracellular amastigote forms cultivated in macrophages. Murine host cells were cultivated for $24 \mathrm{~h}$ and infected with metacyclic promastigotes for $2 \mathrm{~h}$. After $24 \mathrm{~h}$ of infection, AMIO at different concentrations was added to the cultures and treatment maintained for $48 \mathrm{~h}$, with fresh medium with drug added every $24 \mathrm{~h}$. (c) The cytotoxicity of AMIO was also evaluated in noninfected murine macrophages. After treatment with AMIO for $48 \mathrm{~h}$, the macrophages were incubated with Trypan Blue and 400 cells were counted in randomly chosen fields under light microscopy. The experiments were carried out in triplicate, and the bars represent the standard deviation. Statistical analyses were obtained in Prism Software using 2-way ANOVA. The values of $\mathrm{p}$ were obtained comparing the control group with the treated groups: $* * * P<.001$.

the JC-1 fluorochrome, which is a lipophilic cationic mito-chon-dri-al vital dye that becomes concentrated in the mitochondria in response to $\Delta \Psi m$. The dye exists as a monomer at low concentrations, where the emission is $530 \mathrm{~nm}$ (green fluorescence), but at higher concentrations it forms J-aggregates after accumulation in the mitochondrion, where the emission is $590 \mathrm{~nm}$ (red fluorescence). Thus, the fluorescence of JC-1 is considered an indicator of an energized mitochondrial state, and it has been used to measure the $\Delta \Psi m$ in Leishmania [19-21]. Control and AMIO-treated promastigotes after $48 \mathrm{~h}$ of treatment were harvested, washed in $\mathrm{PBS}, \mathrm{pH} 7.2$, added to a reaction medium containing
$125 \mathrm{mM}$ sucrose, $65 \mathrm{mM} \mathrm{KCl,} 10 \mathrm{mM} \mathrm{HEPES} / \mathrm{K}^{+} \mathrm{pH}$ 7.2, $2 \mathrm{mM} \mathrm{Pi}, 1 \mathrm{mM} \mathrm{MgCl} 2$, and $500 \mu \mathrm{M}$ EGTA, and counted in a Neubauer chamber. To evaluate the $\Delta \Psi m$ for each experimental condition, $2.0 \times 10^{7}$ parasites were incubated in $10 \mu \mathrm{g} / \mathrm{mL}$ JC-1 during $30 \mathrm{~min}$, with readings made every 1 min using a Molecular Devices Microplate Reader (a spectrofluorometer SpectraMax M2/M2 ${ }^{\mathrm{e}}$ ). Cells were incubated in the presence of oligomycin $(10 \mu \mathrm{M})$, a F0F1-ATP synthase inhibitor, or FCCP $(1 \mu \mathrm{M})$, a mitochondrial protonophore, during the $30 \mathrm{~min}$ of experiment as positive controls of the depolarization of the mitochondrial membrane. FCCP at the concentration of $2 \mu \mathrm{M}$ was added at the end of all 
experiments to abolish $\Delta \Psi m$. This allowed comparison of the magnitude of $\Delta \Psi m$ under the different experimental conditions. The relative $\Delta \Psi m$ value was obtained calculating the ratio between the reading at $590 \mathrm{~nm}$ and the reading at $530 \mathrm{~nm}$ (590:530 ratio). Control and AMIO-treated promastigotes were also observed under a Zeiss Axioplan epifluorescence microscope using different optical filter sets: (1) for J-aggregate alone, we used $546 \mathrm{~nm}$ band-pass filter for excitation, with a $580 \mathrm{~nm}$ beam splitter, and a $590 \mathrm{~nm}$ long-pass for emission; (2) for monomer and J-aggregate, together, we used a $450-490 \mathrm{~nm}$ for excitation, with a $510 \mathrm{~nm}$ beam splitter, and a $520 \mathrm{~nm}$ long pass for emission. Each experiment was repeated at least three times in triplicate, and the figures shown are representative of these experiments.

2.6. Measurements of ROS Levels. Intracellular ROS level was measured in intact control and AMIO-treated promastigotes as described previously [19]. Briefly, cells $\left(3.0 \times 10^{7}\right)$ were washed, resuspended in $500 \mu \mathrm{L}$ of PBS pH 7.2, and then incubated with the cell-permeable probe green $\mathrm{H}_{2}$ DCFDA at a concentration of $10 \mu \mathrm{g} / \mathrm{mL}$ for $1 \mathrm{~h}$ at $25^{\circ} \mathrm{C}$. After incubation, cells were analyzed at a Molecular Devices Microplate Reader (a spectrofluorometer SpectraMax M2/M2e ${ }^{\mathrm{e}}$, Molecular Devices) using a pair of $507 \mathrm{~nm}$ and $530 \mathrm{~nm}$ as emission and excitation wavelengths, respectively.

2.7. Evaluation of Membrane Integrity and Nile Red Accumulation. Control and AMIO-treated $(6,10$, and $15 \mu \mathrm{M})$ promastigotes were harvested, washed in PBS, $\mathrm{pH} 7.2$, and counted in a Neubauer chamber. After that, cells $(5.0 \times$ $10^{6}$ ) were incubated with $1 \mu \mathrm{M}$ SYTOX Blue and $10 \mu \mathrm{g} / \mathrm{mL}$ Nile Red for 20 minutes. The experiments were made in triplicate, using a black 96-well plate. The final volume in each well was $200 \mu \mathrm{L}$ of cell suspension in PBS. The reading was done in a Molecular Devices Microplate Reader (a spectrofluorometer SpectraMax $\mathrm{M} 2 / \mathrm{M}^{\mathrm{e}}$ ) according to the following wavelengths for excitation and emission, respectively: 444 and $560 \mathrm{~nm}$ for SYTOX Blue, and 485 and $538 \mathrm{~nm}$ for the Nile Red. After the readings, control and AMIO-treated cells were observed under a Zeiss Axioplan epifluorescence microscope equipped with optical filters to SYTOX Blue (the same of DAPI) and Nile Red (450-490 nm for excitation, and $528 \mathrm{~nm}$ for emission). Each experiment was repeated at least three times in triplicate, and the figures shown are representative of these experiments.

2.8. Electron Microscopy. Control and AMIO-treated promastigotes were fixed for $3 \mathrm{~h}$ at $4^{\circ} \mathrm{C}$ in $2.5 \%$ glutaraldehyde (Sigma Chemical Co.) in 0.1M cacodylate buffer ( $\mathrm{pH} 7.2$ ). After fixation, cells were postfixed for $30 \mathrm{~min}$ in a solution containing $1 \% \mathrm{OsO}_{4}$ and $1.25 \%$ potassium ferrocyanide in $0.1 \mathrm{M}$ cacodylate buffer, washed in the same buffer, dehydrated in acetone, and embedded in EPON. Ultrathin sections were stained with uranyl acetate and lead citrate and observed in a Zeiss 900 electron microscope.

\section{Results and Discussion}

3.1. Antiproliferative Effects. L. amazonensis promastigotes were exposed to different concentrations of AMIO, and their proliferation was followed during 4 days. Figure 1(a) shows a concentration-dependent inhibition of the growth induced by the treatment. The $50 \%$ inhibitory concentration $\left(\mathrm{IC}_{50}\right)$ was $4.21 \mu \mathrm{M}$ after $48 \mathrm{~h}$ of treatment. Inhibition of around $100 \%$ was obtained with a concentration of $15 \mu \mathrm{M}$ AMIO, which also induced a delayed lytic effect. We also investigated the effects of AMIO on intracellular amastigotes, the clinically relevant form of the parasite. Macrophages were infected with metacyclic promastigotes and treated with different concentrations of the drug. The $\mathrm{IC}_{50}$ obtained was $0.46 \mu \mathrm{M}$ after $48 \mathrm{~h}$ of treatment, with a total elimination of amastigotes when macrophages were treated with $6 \mu \mathrm{M}$ for $48 \mathrm{~h}$ (Figure 1(b)). Detailed inspection of treated cultures using light microscopy confirmed the potent antiproliferative effect against intracellular amastigotes, as no intact parasites were seen after treatment with concentrations $\geq 6 \mu \mathrm{M}$ of AMIO for $48 \mathrm{~h}$ (Figure 2 ).

Comparing our results with those published for $L$. mexicana [9], another species of the same Leishmania subgenus but that is not relevant for the epidemiology of leishmaniasis in Brazil, the $\mathrm{IC}_{50}$ values found in this study were higher for both stages $(4.21 \mu \mathrm{M}$ for promastigotes and $0.46 \mu \mathrm{M}$ for intracellular amastigotes compared with $900 \mathrm{nM}$ and $8 \mathrm{nM}$, respectively, for L. mexicana), indicating a lower susceptibility of our L. amazonensis strain to the drug. These results are consistent with the known fact that patients infected with $L$. amazonensis are less responsive to the available anti-Leishmania treatments [2]. It is interesting to point out that the clinically relevant intracellular amastigote form of the parasite had a ten-fold higher susceptibility to amiodarone than the extracellular promastigotes, a result similar to those obtained for T. cruzi [8] and L. mexicana [9].

Using the cytotoxicity trypan blue test, viable cells were evaluated in macrophage cultures exposed to AMIO at concentrations varying between 2 and $50 \mu \mathrm{M}$ for $48 \mathrm{~h}$ (Figure 1(c)). The cytotoxicity concentration to reduce $50 \%$ $\left(\mathrm{CC}_{50}\right)$ of viable macrophages was $12.9 \mu \mathrm{M}$ (Figure 1), giving a mean selectivity index of 28 . Light microscopy showed that macrophages treated with 6 and $8 \mu \mathrm{M}$ AMIO did not present any significant alteration (Figure 2 ). In several mammalian cell lines, AMIO has been described to be very toxic [17, 22, 23]. However, the selectivity of AMIO for trypanosomatid parasites over mammalian cells and organisms has been confirmed in experimental models of Chagas' disease and leishmaniasis [8-10] and more recently in clinical studies $[24,25]$.

\subsection{Effects of AMIO on the Neutral Lipid Accumulation and on} the Fine Structure of L. amazonensis Promastigotes. In order to evaluate the effect of AMIO on the plasma membrane integrity and the presence of lipid-storage bodies in $L$. amazonensis promastigotes, we performed a quantitative analysis after simultaneous incubation of the cells with SYTOX Blue and Nile Red. The results indicated that at the higher concentration used (15 $\mu \mathrm{M}$ AMIO), the plasma 


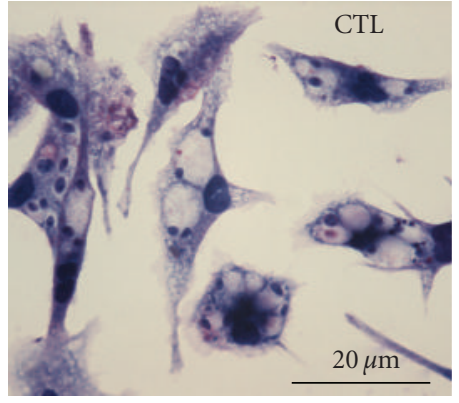

(a)

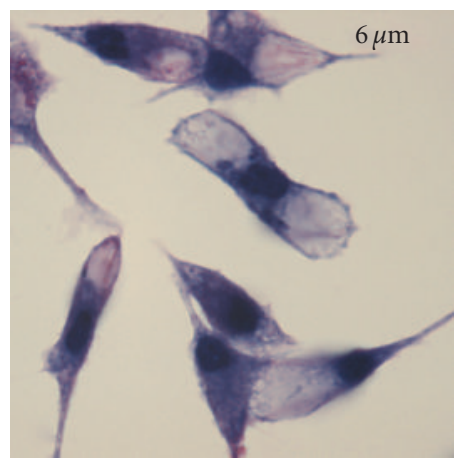

(c)

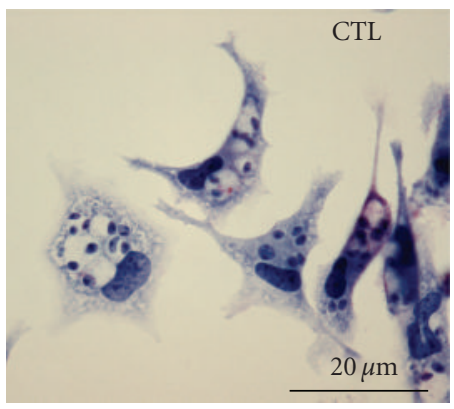

(e)

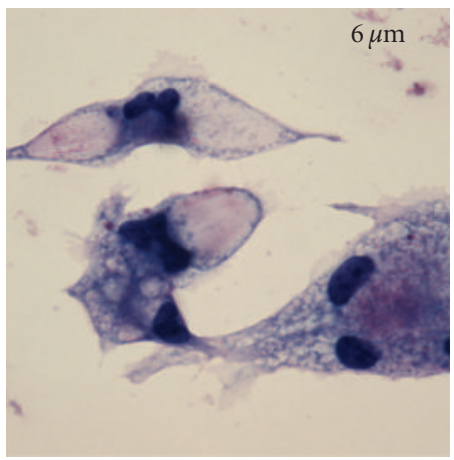

(g)

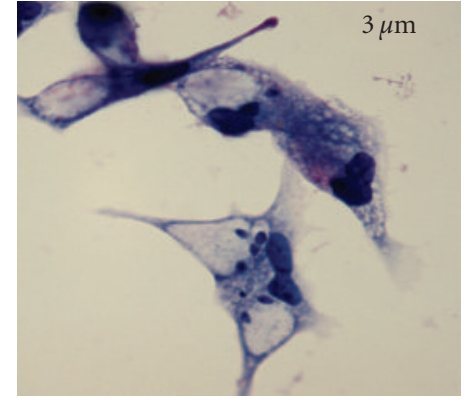

(b)

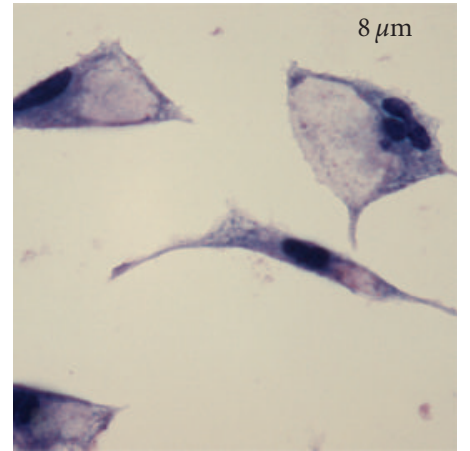

(d)

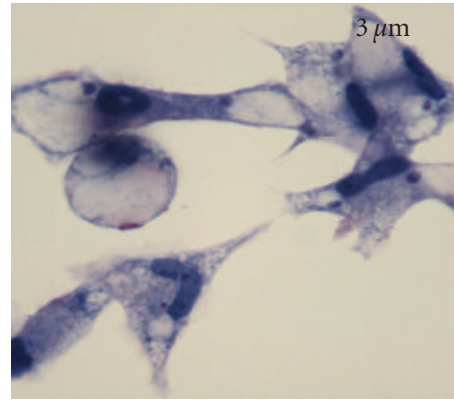

(f)

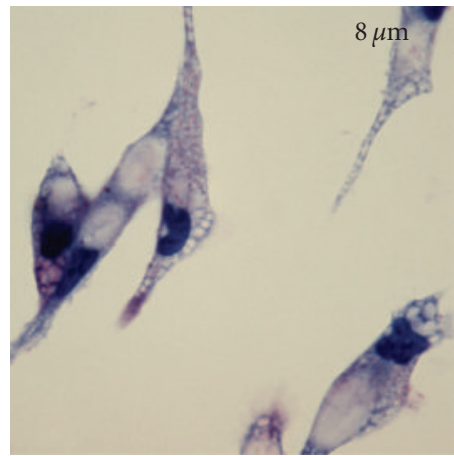

(h)

FIGURE 2: Light microscopy of intracellular amastigote forms of Leishmania amazonensis treated with different concentrations of amiodarone (AMIO). (a-d) After $24 \mathrm{~h}$ of treatment, it is possible to observe a significant reduction in the parasite number with 6 and $8 \mu \mathrm{M}$ AMIO (c, d). $(\mathrm{e}-\mathrm{h})$ After $48 \mathrm{~h}$ of treatment, a significant reduction was observed in the cultures treated with $3 \mu \mathrm{M}$ AMIO (f). Infection of macrophages was carried out as described in Materials and Methods and Figure 1. For all the images, the scale bars are similar to those observed in the panels (a) and (e). (a) Control parasites/24 h of treatment, which means $48 \mathrm{~h}$ of infection; (e) Control parasites/48 h of treatment, which means $72 \mathrm{~h}$ of infection. 


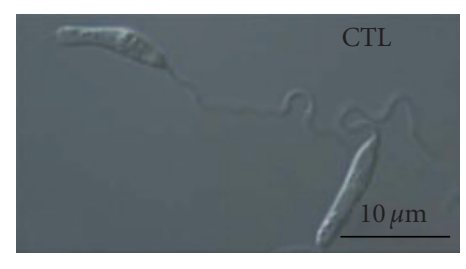

(a)

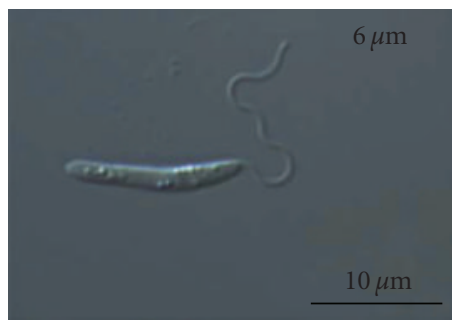

(c)

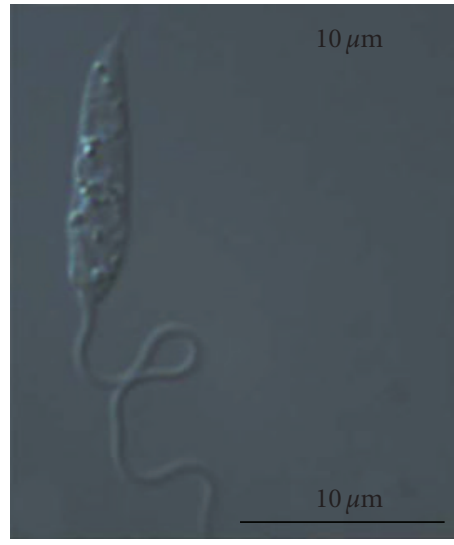

(e)

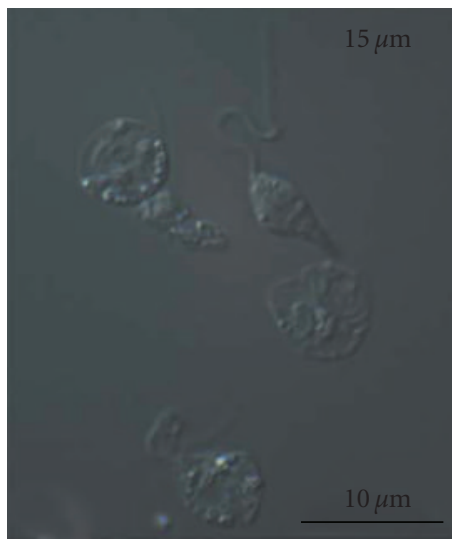

(g)

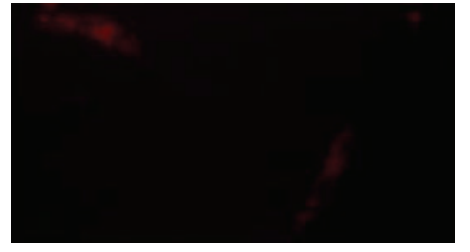

(b)

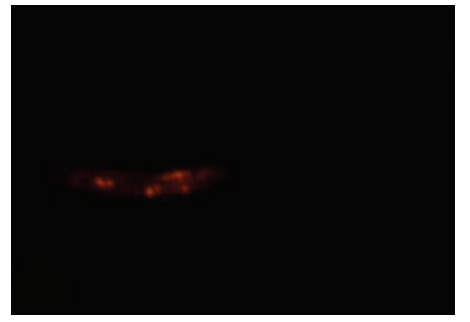

(d)

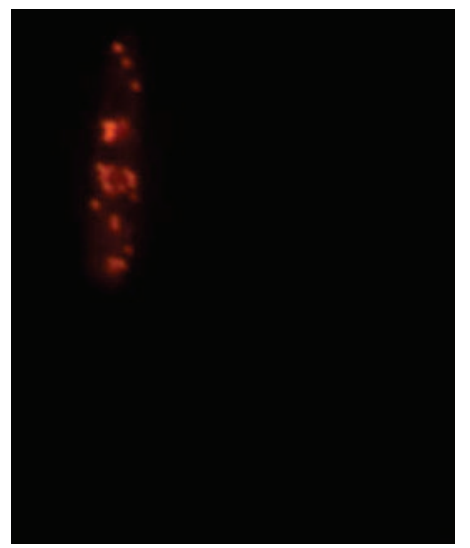

(f)

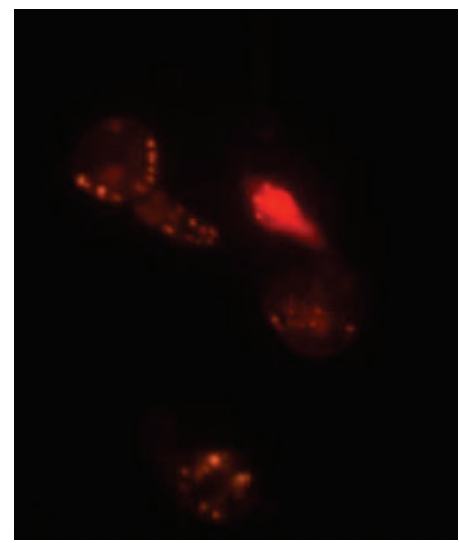

(h)

Figure 3: Differential interference contrast microscopy (DIC) and fluorescence microscopy with Nile Red of Leishmania amazonensis promastigotes untreated (a-b) and treated with different concentrations of AMIO $(6,10$ and $15 \mu \mathrm{M})$ for $48 \mathrm{~h}(\mathrm{c}-\mathrm{h}$, resp.). In the treated promastigotes, images suggest an accumulation of lipid-storage bodies in the cytoplasm, which is concentration dependent. In (g), parasites treated with $15 \mu \mathrm{M}$ AMIO are completely modified. The conditions used to harvest the parasites, to incubate with Nile Red and to observe under the microscopy using the correct filters sets are described in Materials and Methods. 


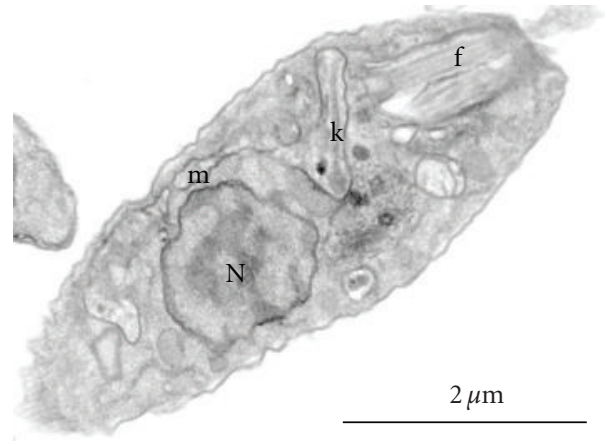

(a)

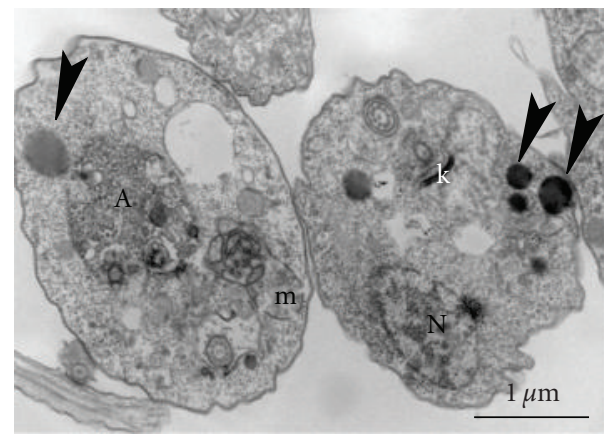

(c)

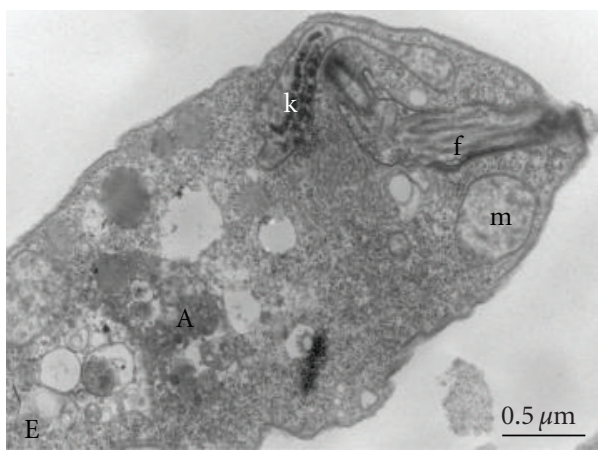

(e)

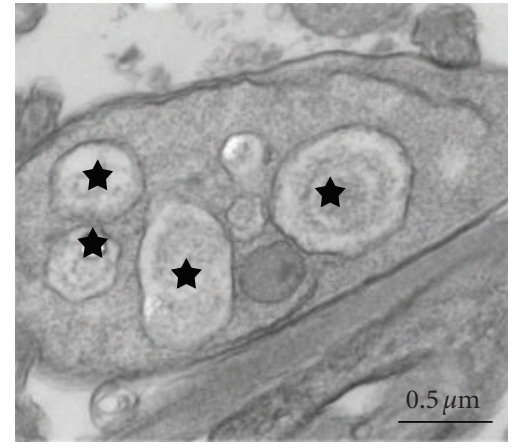

(b)

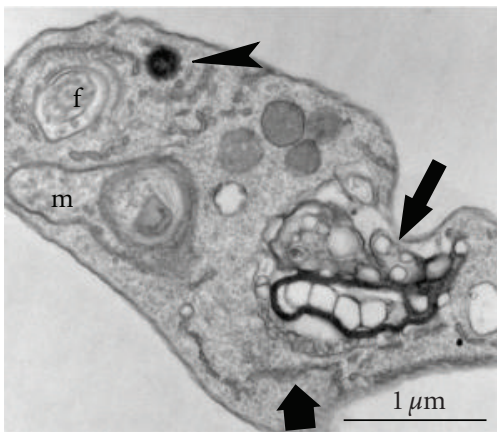

(d)

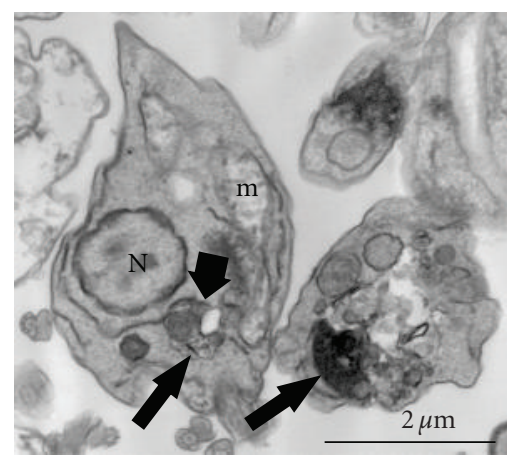

(f)

FIGURE 4: Different ultrastructural alterations on Leishmania amazonensis promastigotes induced by the treatment with amiodarone (AMIO). (a) Ultrathin section of L. amazonensis promastigotes without treatment, which presents a normal ultrastructure of organelles such as (mitochondrion) $\mathrm{m}$, (kinetoplast) $\mathrm{k}$, (nucleus) $\mathrm{N}$ and (flagellum) $\mathrm{f}$. (b) Electron micrograph of L. amazonensis treated with $5 \mu \mathrm{M}$ AMIO for $48 \mathrm{~h}$ presenting many vacuoles similar to autophagosomes (stars). (c-e) After treatment with $15 \mu \mathrm{M}$ AMIO for $24 \mathrm{~h}$, it is possible to observe the presence of large autophagosomes a associated with endoplasmic reticulum profiles (big arrow), lipid bodies (arrowheads), and alterations in the mitochondrion-kinetoplast complex and chromatin condensation. (f) Promastigotes treated with $20 \mu \mathrm{M}$ AMIO for $24 \mathrm{~h}$ presented drastic alterations and destruction of the cytoplasm, where it is possible to observe the presence of autophagosomes (arrows) sometimes associated with endoplasmic reticulum profile (big arrow). A: autophagosome; f: flagellum; k: kinetoplast; m: mitochondrion; N: nucleus.

membrane permeability was significantly altered (data not shown). This effect was not observed at lower concentrations (6 and $10 \mu \mathrm{M}$ AMIO).

On the other hand, incubation with Nile Red revealed a concentration-dependent effect on neutral lipids' accumulation, which was quantified by fluorimeter (data not shown). The visualization of AMIO-treated promastigotes under fluorescence microscopy revealed the presence of many lipid bodies positive to Nile Red and randomly distributed throughout the cytoplasm (Figures 3(a)-3(h)). The fluorescence images indicate a concentration-dependent increase in the number of lipid bodies (Figures 3(d), 3(f), and $3(\mathrm{~h})$ ), while differential interference contrast microscopy (DIC) revealed an important alteration in the shape of 


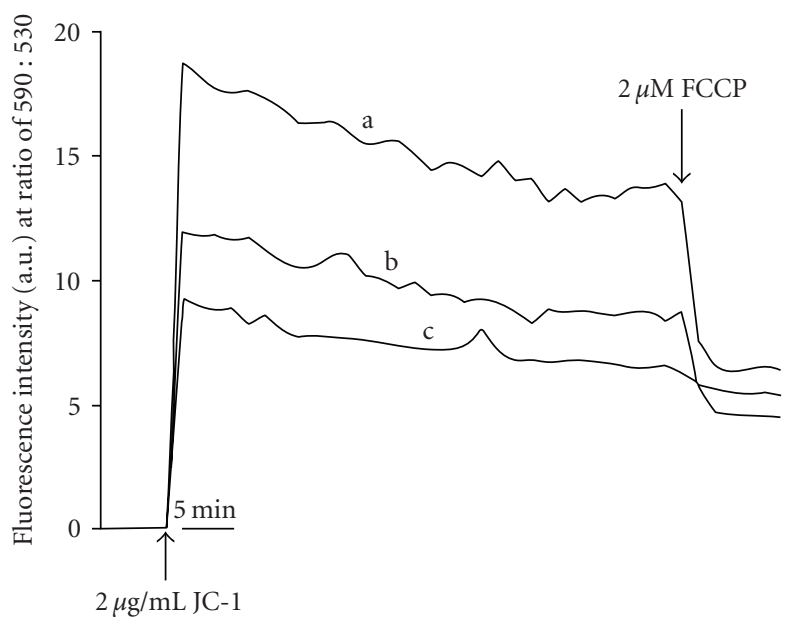

(a)

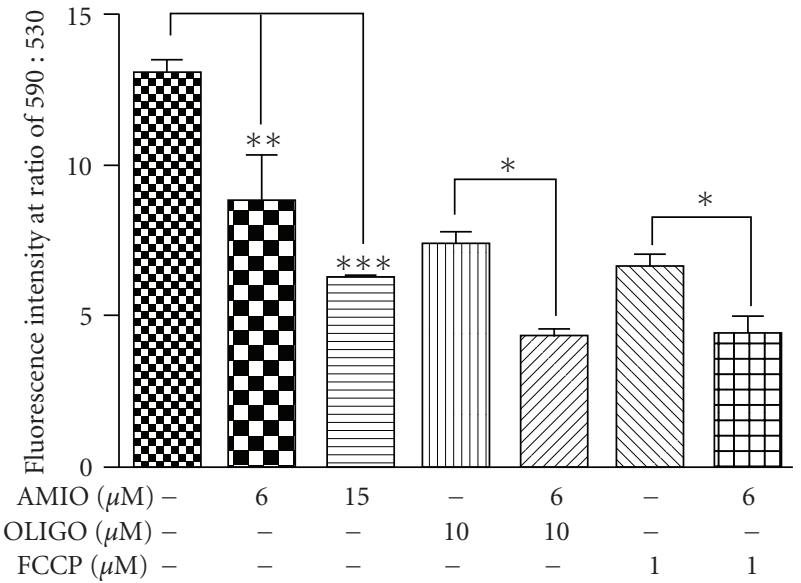

(b)

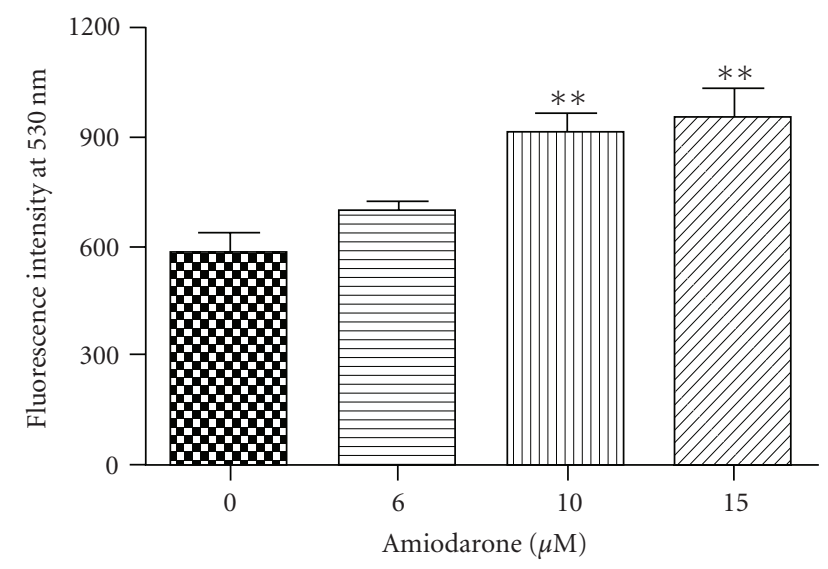

(c)

FIGURE 5: Effects of the AMIO on the mitochondrial function of Leishmania amazonensis promastigotes. (a) Values of $\Delta \Psi m$ were evaluated during $30 \mathrm{~min}$, after which the uncoupler FCCP was added to collapse mitochondrial potential. Cells grown in the presence of two concentrations of amiodarone, 6 and $15 \mu \mathrm{M}$, for $48 \mathrm{~h}$ (curves b and c, resp.) were observed. (b) Effect of oligomycin (10 $\mu \mathrm{M})$ and FCCP $(1 \mu \mathrm{M})$ in the $\Delta \Psi m$ was also evaluated. (c) Production of ROS was measured in the same cells. Control and treated cells were incubated with $\mathrm{H}_{2}$ DCFDA, and the fluorescence intensity was measured in a Microplate Reader. Fluorescence intensity is expressed as arbitrary units (A.U.). The experiments were performed three times, each time in triplicate, and the figures shown are representative of these experiments.

promastigotes treated with $15 \mu \mathrm{M}$ AMIO, which appeared rounded and swollen with a clear evidence of loss of the cytoplasmatic content (Figure $3(\mathrm{~g})$ ), confirming the alteration on the plasma membrane integrity indicated by SYTOX Blue staining. These results are consistent with the reported effect of AMIO on the de novo sterol biosynthesis in T. cruzi [8] and L. mexicana [9]. Similar results were obtained with $L$. amazonensis treated with squalene synthase inhibitors [26].

We also evaluated the effect of AMIO on the fine structure of L. amazonensis promastigote. Figure 4(a) shows a longitudinal section of a control promastigote presenting different organelles such as mitochondrion, nucleus, kinetoplast, and flagellum with normal ultrastructure. Parasites incubated in the presence of AMIO displayed significant morphological changes, which varied from discrete alterations to a total destruction of the parasite, depending on the drug concentration and length of incubation. The changes began to appear with $5 \mu \mathrm{M}$ AMIO after $48 \mathrm{~h}$ of incubation, when it was possible to observe the presence of some vacuoles similar to autophagosomes (Figure 4(b), stars). With just $24 \mathrm{~h}$ of treatment, but using higher concentrations (15 and $20 \mu \mathrm{M}$ ), several alterations could be observed such as presence of lipid-storage bodies (arrowheads), large myelin-like figures (arrow) with presence of endoplasmic reticulum profiles (big arrow), and autophagic structures containing cellular debris (Figures 4(c)-4(f)). These structures could be related to a degradation of damaged organelle induced by the drug treatment. In addition, alterations in the mitochondrion, in the kinetoplast, and in the chromatin condensation can be observed in Figures 4(c)-4(d). We also observed the presence of several small vesicles inside the flagellar pocket (Figure 4(e)) and many cells swollen and completely destroyed after treatment with 15 and $20 \mu \mathrm{M}$ AMIO for $24 \mathrm{~h}$ (Figure $4(\mathrm{f}))$. All these alterations are characteristic of the 


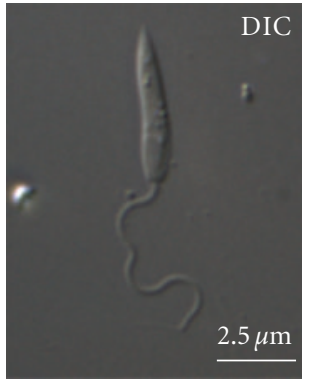

(a)

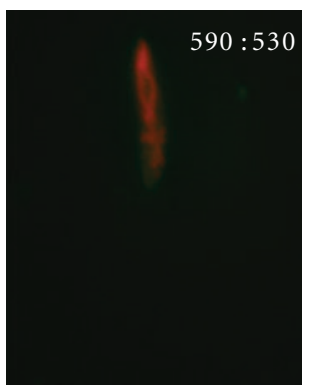

(b)

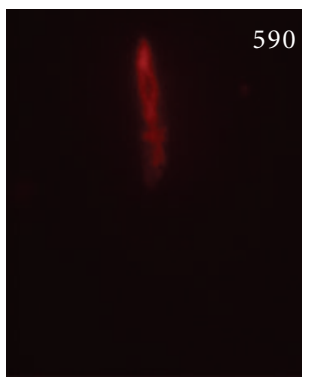

(c)

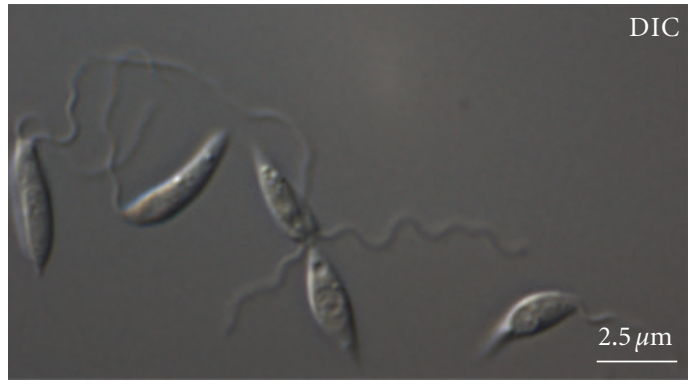

(d)

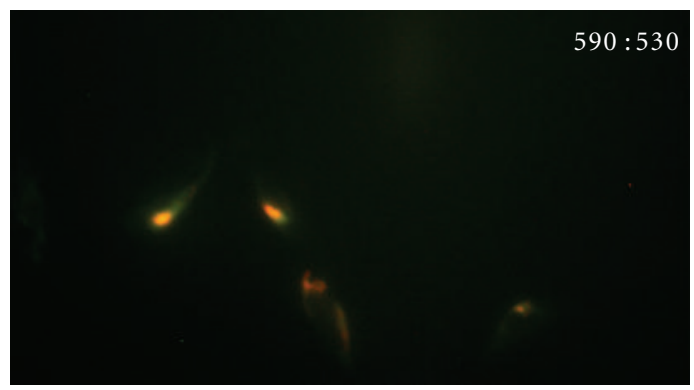

(e)

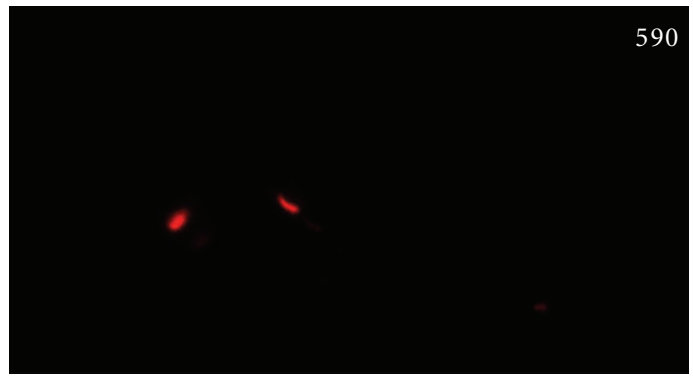

(f)

FIGURE 6: Differential interference contrast (DIC) microscopy and fluorescence microscopy with JC-1 of Leishmania amazonensis promastigotes untreated $(\mathrm{a}-\mathrm{c})$ and treated with $15 \mu \mathrm{M}$ AMIO for $48 \mathrm{~h}(\mathrm{~d}-\mathrm{f})$. In panels (b) and (c), the accumulation of aggregated-JC1 is observed in the whole extension of the control mitochondrion. In cells treated with $15 \mu \mathrm{M}$ AMIO (e-f), the accumulation of J-aggregates occurs in some portions of the mitochondrion, indicating a partial dissipation of the $\Delta \Psi m$. Panels (b) and (e) show an image of the monomers and J-aggregates together, while panels (c) and (f) show the image of only J-aggregates.

three main types of cell death: apoptosis, necrosis, and autophagy (reviewed in [27]).

Some of the lipid bodies appeared near the plasma membrane and the autophagic-like structures, which could be related, respectively, to alterations of the biophysical properties of the plasma membrane and degradation of abnormal lipids that accumulated as a consequence of the treatment. Thus, alterations of lipid composition resulting from treatment with AMIO could interfere with plasma membrane integrity, leading to cell death by necrosis.

\subsection{Effects of AMIO on the Mitochondrial Physiology and} Ultrastructure of L. amazonensis Promastigotes. We investigated the effect of AMIO on the mitochondrial function and ultrastructure using three criteria: mitochondrial transmembrane electric potential $(\Delta \Psi m)$ using JC-1 fluorochrome, production of reactive oxygen species (ROS) using a green
$\mathrm{H}_{2}$ DCFDA probe, and transmission electron microscopy. JC1 is a cell-permeant cationic lipophilic fluorochrome that accumulates in the functional mitochondrion forming redfluorescent J-aggregates. On the other hand, mitochondrial de-energization leads to an accumulation of green fluorescence monomers. Thus, the decrease in the red/green fluorescence intensity ratio indicates a collapse in the mitochondrial transmembrane potential. There are some advantages of using the JC-1: (1) it is not necessary to permeabilize the cells; (2) it is easy to quantify in a fluorimeter as well as to observe the process under a fluorescence microscopy. Promastigotes were treated with $0,6,10$, and $15 \mu \mathrm{M}$ AMIO for $48 \mathrm{~h}$ prior to the analysis of the mitochondrial features. Incubation with JC-1 for 30 min indicated that cells treated with 6 and $15 \mu \mathrm{M}$ AMIO had a very significant reduction of $\Delta \Psi m$ (Figure $5(\mathrm{a})$, traces $\mathrm{b}$ and $\mathrm{c}$ ) when compared with the control (untreated) parasites (Figure 5(a), trace a). The results indicated a marked reduction in the mitochondrial 


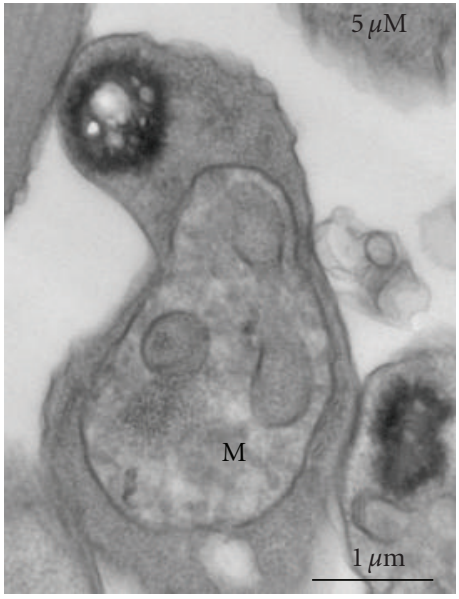

(a)

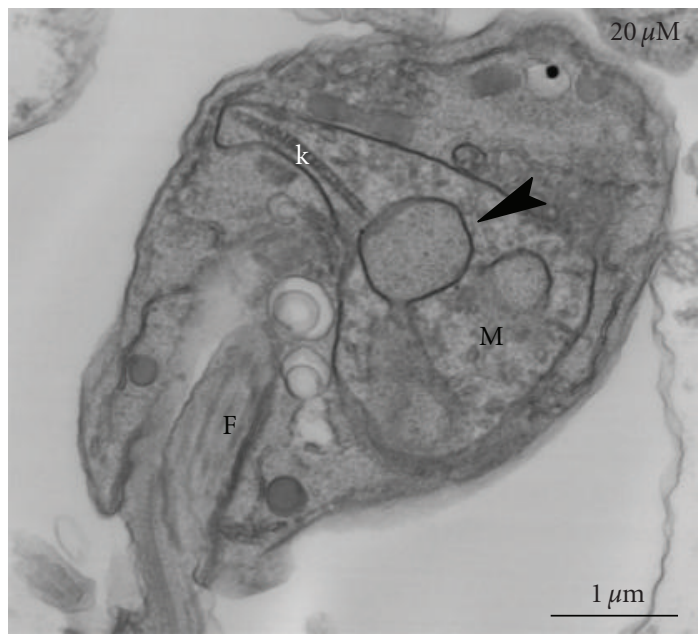

(d)

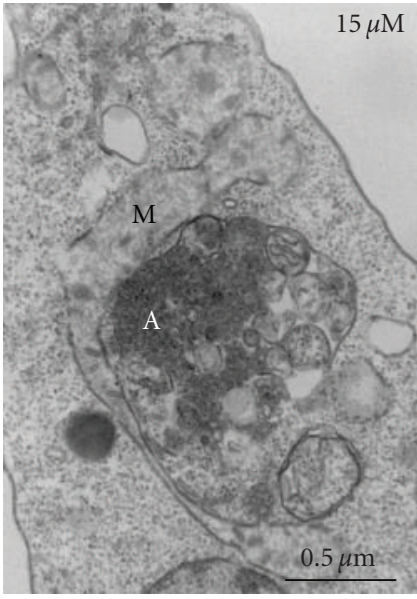

(b)

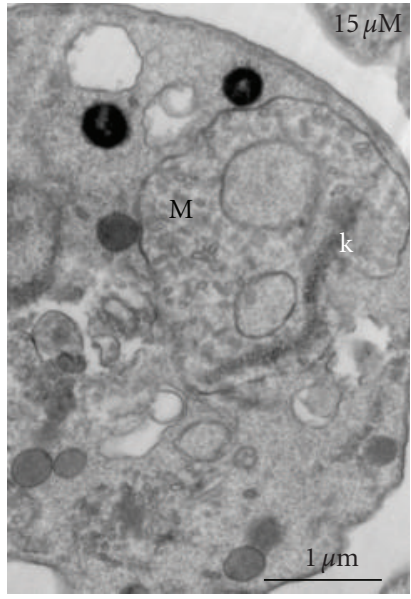

(c)

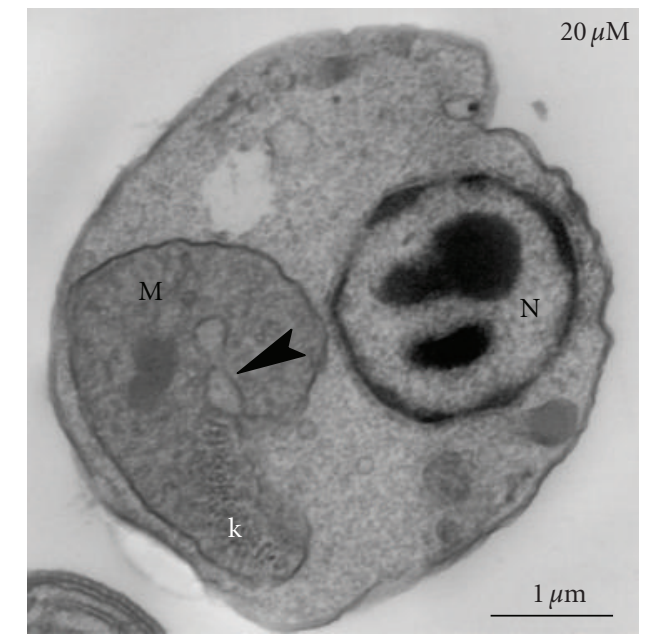

(e)

FIGURE 7: Ultrathin sections of L. amazonensis promastigotes treated with different concentrations of AMIO showing several alterations in the mitochondrial ultrastructure such as marked swelling ( $a, c, d$, and e) with loss of the matrix content (a), alterations in the mitochondrial membrane (arrowheads), and presence of autophagic structures near a modified mitochondrion (b). A: autophagic structure; F: flagellum; k: kinetoplast; M: mitochondrion, N: nucleus.

polarization with $6 \mu \mathrm{M}$ AMIO (Figure 5(a), trace b), and an almost total depolarization of the mitochondrial membrane potential with $15 \mu \mathrm{M}$ AMIO (Figure 5(a), trace c). After 30 min of JC-1 uptake, $2 \mu \mathrm{M}$ FCCP was added to fully collapse the $\Delta \Psi m$ : it can be seen that in control parasites the release of JC-1 was more prominent than in treated promastigotes. To compare these findings with other situations that can induce depolarization of the mitochondrion, control and treated parasites were incubated with two classical inhibitors of the mitochondrial function: olygomicin, which is an inhibitor of the F0F1-ATP synthase, and FCCP, a classical protonophore uncoupler that dissipates the mitochondrial electrochemical $\mathrm{H}+$ gradient (Figure 5(b)). It can be seen that the reduction of $\Delta \Psi m$ found in cells grown in the presence of 6 and $15 \mu \mathrm{M}$ AMIO (Figure 5(a)) is similar to those obtained in control cells incubated with olygomicin or FCCP (Figure 5(b)). In addition, when these inhibitors were added in AMIO-treated promastigotes, the decrease of the $\Delta \Psi m$ was more evident.

We also investigated the effect of AMIO on the production of reactive oxygen species (ROS), as it is known that inhibition of oxidative phosphorylation induces an increase in the production of ROS. The results shown in Figure 5(c) indicate that cells grown in the presence of AMIO at the same concentrations used to evaluate the $\Delta \Psi m$, showed very significant and concentration-dependent increase in the production of ROS. The effect was most evident with 10 and $15 \mu \mathrm{M}$ AMIO.

Alterations of the mitochondrion were also investigated using fluorescence and transmission electron microscopy, confirming the results obtained at the fluorimeter. Visualization of control and AMIO-treated promastigotes incubated with JC-1 under fluorescence microscopy revealed 
that cells grown with $15 \mu \mathrm{M}$ AMIO for $48 \mathrm{~h}$ showed reduced accumulation of the fluorochrome, restricted to the kinetoplast region of the single giant mitochondrion (Figures 6(e) and 6(f)), indicating a loss of only $\Delta \Psi m$ in most parts of the organelle. In contrast, in control promastigotes, JC-1 accumulated in the whole extension of the ramified mitochondrion (Figures 6(b) and 6(c)). Images (b) and (e) show the fluorescence for monomers and Jaggregates, together, while the images (c) and (f) show the fluorescence for J-aggregates alone.

Finally, ultrastructural alterations on the mitochondrion were also investigated: in Figure 7 the occurrence of dramatic modifications in different aspects of the organelle can be seen. It is evident that long-term incubation with AMIO induced mitochondrial swelling at all the concentrations tested (Figures 7(a), 7(c), 7(d) and 7(e)), which is followed by alterations in the mitochondrial membranes (Figures 7(d) and 7(e), arrowheads), appearance of circular cristae (Figure 7(a) and 7(c)), and loss of the mitochondrial matrix content (Figures $7(\mathrm{a})$ and $7(\mathrm{~d})$ ). In addition, we also observed an important interaction between the mitochondrion and structures similar to autophagic vacuoles (Figure $7(\mathrm{c})$ ). In this same figure, alterations in the kinetoplast structure are also evident. Ultrastructural alterations on the mitochondrion also predominate in T. cruzi and Leishmania cells treated with sterol biosynthesis inhibitors, including quinuclidine derivatives, azoles and azasterols, indicating that this organelle is an important target for compounds that interfere with sterol and lipid composition [27-35].

The dose-dependent reduction of $\Delta \Psi m$ induced by amiodarone was correlated with the increase in the production of reactive oxygen species and mitochondrial ultrastructural alterations detected with transmission electron microscopy. Two possible explanations for these combined alterations are: (1) Calcium release from the mitochondrion, which would result from a direct action of AMIO on the organelle and lead to apoptotic cell death [36]; and, (2) Sterol biosynthesis inhibition, as observed in fungi [37] and trypanosomatids $[8,9]$, leading to an important alteration in the lipid composition of the mitochondrial membranes that should modify their biophysical properties [28, 38, 39], and loss of mitochondrial function.

\section{Conclusion}

In conclusion, the results of this work support the potential usefulness of AMIO as a chemotherapeutic agent against Leishmania amazonensis. Such activity is mainly mediated by profound and selective effects on the ultrastructure and physiology of the parasite mitochondrion, which culminate in cell death by necrosis, apoptosis, or autophagy. More studies are necessary to better characterize the different types of cell death associated with the mechanisms of action of AMIO and the activity of the drug in murine models of cutaneous leishmaniasis by L. amazonensis.

\section{Acknowledgments}

This work was supported by Conselho Nacional de Desenvolvimento Científico (CNPq), Fundação Carlos Chagas Filho de Amparo à Pesquisa do Estado do Rio de Janeiro (FAPERJ), Coordenação de Aperfeiçoamento de Pessoal de Nível Superior (CAPES), and Programas de Núcleos de Excelência (PRONEX). J. C. F. Rodrigues and S. T. M. Silva thank Joseane Lima Prado Godinho for helpful discussions.

\section{References}

[1] P. Desjeux, "Leishmaniasis: current situation and new perspectives," Comparative Immunology, Microbiology and Infectious Diseases, vol. 27, no. 5, pp. 305-318, 2004.

[2] P. D. Marsden and T. C. Jones, "Clinical manifestations, diagnosis, and treatment of leishmaniasis," in Human Parasitic Diseases, Leishmaniasis, K. P. Chang and R. S. Bray, Eds., vol. 1, pp. 183-198, Elsevier Science, New York, NY, USA, 1985.

[3] S. L. Croft, S. Sundar, and A. H. Fairlamb, "Drug resistance in leishmaniasis," Clinical Microbiology Reviews, vol. 19, no. 1, pp. 111-126, 2006.

[4] S. L. Croft, M. P. Barrett, and J. A. Urbina, "Chemotherapy of trypanosomiases and leishmaniasis," Trends in Parasitology, vol. 21, no. 11, pp. 508-512, 2005.

[5] H. Sindermann, S. L. Croft, K. R. Engel et al., "Miltefosine (Impavido): the first oral treatment against leishmaniasis," Medical Microbiology and Immunology, vol. 193, no. 4, pp. 173-180, 2004.

[6] N. K. Ganguly, "Oral miltefosine may revolutionize treatment of visceral leishmaniasis. The potential impact of miltefosine on visceral leishmaniasis in India," TDR News, no. 68, p. 2, 2002.

[7] J. van Griensven, M. Balasegaram, F. Meheus, J. Alvar, L. Lynen, and M. Boelaert, "Combination therapy for visceral leishmaniasis," The Lancet Infectious Diseases, vol. 10, no. 3, pp. 184-194, 2010.

[8] G. Benaim, J. M. Sanders, Y. García-Marchan et al., "Amiodarone has intrinsic anti-Trypanosoma cruzi activity and acts synergistically with posaconazole," Journal of Medicinal Chemistry, vol. 49, no. 3, pp. 892-899, 2006.

[9] X. Serrano-Martín, Y. García-Marchan, A. Fernandez et al., "Amiodarone desestabilizes intracellular $\mathrm{Ca}^{2+}$ homeostasis and biosynthesis of sterols in Leishmania mexicana," Antimicrobial Agents and Chemotherapy, vol. 53, pp. 1403-1410, 2009.

[10] X. Serrano-Martín, G. Payares, M. de Lucca, J. C. Martinez, A. Mendoza-León, and G. Benaim, "Amiodarone and miltefosine act synergistically against Leishmania mexicana and can induce parasitological cure in a murine model of cutaneous leishmaniasis," Antimicrobial Agents and Chemotherapy, vol. 53, no. 12, pp. 5108-5113, 2009.

[11] W. E. Courchesne, "Characterization of a novel, broad-based fungicidal activity for the antiarrhythmic drug amiodarone," Journal of Pharmacology and Experimental Therapeutics, vol. 300, no. 1, pp. 195-199, 2002.

[12] S. S. Gupta, V.-K. Ton, V. Beaudry, S. Rulli, K. Cunningham, and R. Rao, "Antifungal activity of amiodarone is mediated by disruption of calcium homeostasis," Journal of Biological Chemistry, vol. 278, no. 31, pp. 28831-28839, 2003.

[13] A. I. Pozniakovsky, D. A. Knorre, O. V. Markova, A. A. Hyman, V. P. Skulachev, and F. F. Severin, "Role of mitochondria in the pheromone- and amiodarone-induced programmed death 
of yeast," Journal of Cell Biology, vol. 168, no. 2, pp. 257-269, 2005.

[14] N. Dzimiri and A. A. Almotrefi, "Actions of amiodarone on mitochondrial ATPase and lactate dehydrogenase activities in guinea pig heart preparations," European Journal of Pharmacology, vol. 242, no. 2, pp. 113-118, 1993.

[15] S. M. Ribeiro, A. P. Campello, A. J. Nascimento, and M. L. Kluppel, "Effect of amiodarone (AMD) on the antioxidant enzymes, lipid peroxidation and mitochondrial metabolism," Cell Biochemistry and Function, vol. 15, no. 3, pp. 145-152, 1997.

[16] J. W. Card, B. R. Lalonde, E. Rafeiro et al., "Amiodaroneinduced disruption of hamster lung and liver mitochondrial function: lack of association with thiobarbituric acid-reactive substance production," Toxicology Letters, vol. 98, no. 1-2, pp. 41-50, 1998.

[17] G. Varbiro, A. Toth, A. Tapodi, B. Veres, B. Sumegi, and F. Gallyas, "Concentration dependent mitochondrial effect of amiodarone," Biochemical Pharmacology, vol. 65, no. 7, pp. 1115-1128, 2003.

[18] L. G. Warren, "Metabolism of Schizotrypanum cruzi Chagas. I. Effect of culture age and substrate concentration on respiratory rate," Journal of Parasitology, vol. 46, pp. 529-539, 1960.

[19] N. Sen, B. B. Das, A. Ganguly, T. Mukherjee, S. Bandyopadhyay, and H. K. Majumder, "Camptothecin-induced imbalance in intracellular cation homeostasis regulates programmed cell death in unicellular hemoflagellate Leishmania donovani," Journal of Biological Chemistry, vol. 279, no. 50, pp. 52366-52375, 2004.

[20] N. Sen, B. B. Das, A. Ganguly et al., "Camptothecin induced mitochondrial dysfunction leading to programmed cell death in unicellular hemoflagellate Leishmania donovani," Cell Death and Differentiation, vol. 11, no. 8, pp. 924-936, 2004.

[21] A. Roy, A. Ganguly, S. BoseDasgupta et al., "Mitochondriadependent reactive oxygen species-mediated programmed cell death induced by 3,3'-diindolylmethane through inhibition of F0F1-ATP synthase in unicellular protozoan parasite Leishmania donovani," Molecular Pharmacology, vol. 74, no. 5, pp. 1292-1307, 2008.

[22] R. Hofmann, C. Steinwender, J. Kammler, A. Kypta, and F. Leisch, "Effects of a high dose intravenous bolus amiodarone in patients with atrial fibrillation and a rapid ventricular rate," International Journal of Cardiology, vol. 110, no. 1, pp. 27-32, 2006.

[23] E. E. Golli-Bennour, A. Bouslimi, O. Zouaoui, S. Nouira, A. Achour, and H. Bacha, "Cytotoxicity effects ofamiodarone on cultured cells," Experimental and Toxicologic Pathology. In press.

[24] A. E. Paniz-Mondolfi, A. M. Pérez-Álvarez, O. Reyes-Jaimes et al., "Concurrent Chagas' disease and borderline disseminated cutaneous leishmaniasis: the role of amiodarone as an antitrypanosomatidae drug," Therapeutics and Clinical Risk Management, vol. 4, no. 3, pp. 659-663, 2008.

[25] A. E. Paniz-Mondolfi, A. M. Pérez-Álvarez, G. Lanza, E. Márquez, and J. L. Concepción, "Amiodarone and itraconazole: a rational therapeutic approach for the treatment of chronic Chagas' disease," Chemotherapy, vol. 55, no. 4, pp. 228-233, 2009.

[26] J. C. F. Rodrigues, J. L. Concepcion, C. Rodrigues, A. Caldera, J. A. Urbina, and W. de Souza, "In vitro activities of ER-119884 and E5700, two potent squalene synthase inhibitors, against Leishmania amazonensis: antiproliferative, biochemical, and ultrastructural effects," Antimicrobial Agents and Chemotherapy, vol. 52, no. 11, pp. 4098-4114, 2008.

[27] J. C. F. Rodrigues and W. de Souza, "Ultrastructural alterations in organelles of parasitic protozoa induced by different classes of metabolic inhibitos," Current Pharmaceutical Design, vol. 14, no. 9, pp. 925-938, 2008.

[28] J. C. F. Rodrigues, C. F. Bernardes, G. Visbal, J. A. Urbina, A. E. Vercesi, and W. de Souza, "Sterol methenyl transferase inhibitors alter the ultrastructure and function of the Leishmania amazonensis mitochondrion leading to potent growth inhibition," Protist, vol. 158, no. 4, pp. 447-456, 2007.

[29] M. A. Vannier-Santos, J. A. Urbina, A. Martiny, A. Neves, and W. de Souza, "Alterations induced by the antifungal compounds ketoconazole and terbinafine in Leishmania," Journal of Eukaryotic Microbiology, vol. 42, no. 4, pp. 337-346, 1995.

[30] J. Vivas, J. A. Urbina, and W. de Souza, "Ultrastructural alterations in Trypanosoma (Schizotrypanum) cruzi induced by $\Delta^{24(25)}$-sterol methyltransferase inhibitors and their combinations with ketoconazole," International Journal of Antimicrobial Agents, vol. 7, pp. 235-240, 1996.

[31] J. C. F. Rodrigues, M. Attias, C. Rodriguez, J. A. Urbina, and W. de Souza, "Ultrastructural and biochemical alterations induced by 22,26 -azasterol, a $\Delta^{24(25)}$-sterol methyltransferase inhibitors, on promastigote and amastigote forms of Leishmania amazonensis," Antimicrobial Agents and Chemotherapy, vol. 46, no. 2, pp. 487-499, 2002.

[32] F. Magaraci, C. J. Jimenez, C. Rodrigues et al., "Azasterols as inhibitors of sterol 24-methyltransferase in Leishmania species and Trypanosoma cruzi," Journal of Medicinal Chemistry, vol. 46, no. 22, pp. 4714-4727, 2003.

[33] S. O. Lorente, J. C. F. Rodrigues, C. J. Jiménez et al., "Novel azasterols as potential agents for treatment of Leishmaniasis and Trypanosomiasis," Antimicrobial Agents and Chemotherapy, vol. 48, no. 8, pp. 2937-2950, 2004.

[34] A. C. Granthon, M. V. Braga, J. C. F. Rodrigues et al., "Alterations on the growth and ultrastructure of Leishmania chagasi induced by squalene synthase inhibitors," Veterinary Parasitology, vol. 146, no. 1-2, pp. 25-34, 2007.

[35] W. de Souza and J. C. F. Rodrigues, "Sterol biosynthesis pathway as target for anti-trypanosomatid drugs," Interdisciplinary Perspectives on Infectious Diseases, vol. 2009, Article ID 642502, 19 pages, 2009.

[36] W. E. Courchesne and S. Ozturk, "Amiodarone induces a caffeine-inhibited, MID1-depedent rise in free cytoplasmic calcium in Saccharomyces cerevisiae," Molecular Microbiology, vol. 47, no. 1, pp. 223-234, 2003.

[37] E. Krajewska-Kulak and W. Niczyporuk, "Effects of the combination of ketoconazole and calcium channel antagonists against Candida albicans in vitro," Arzneimittel Forschung, vol. 43, no. 7, pp. 782-783, 1993.

[38] C. O. Rodrigues, R. Catisti, S. A. Uyemura et al., "The sterol composition of Trypanosoma cruzi changes after growth in different culture media and results in different sensitivity to digitonin-permeabilization," Journal of Eukaryotic Microbiology, vol. 48, no. 5, pp. 588-594, 2001.

[39] I. V. Palmié-Peixoto, M. R. Rocha, J. A. Urbina, W. de Souza, M. Einicker-Lamas, and M. C. M. Motta, "Effects of sterol biosynthesis inhibitors on endosymbiont-bearing try panosomatids," FEMS Microbiology Letters, vol. 255, no. 1, pp. 33-42, 2006. 

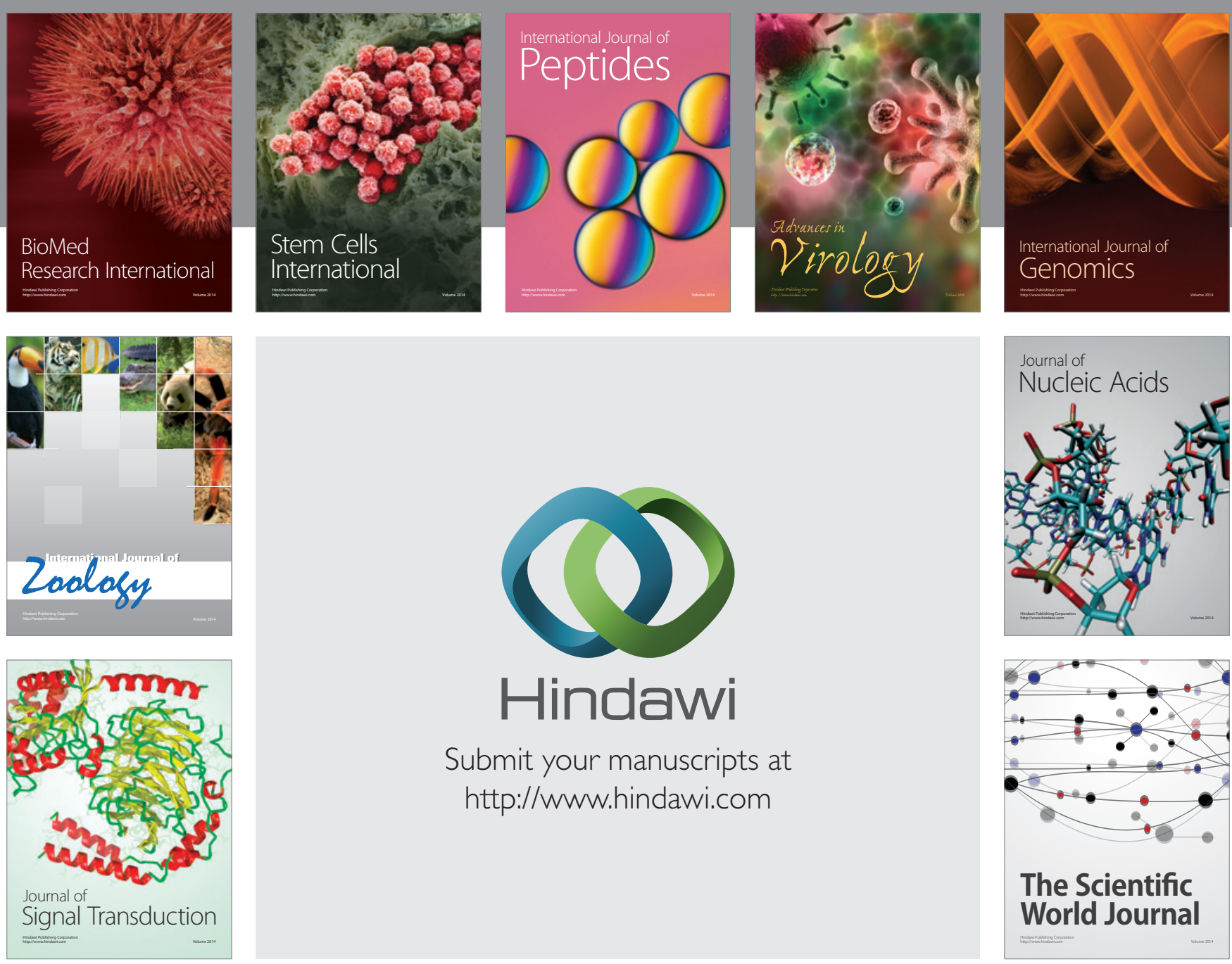

Submit your manuscripts at

http://www.hindawi.com
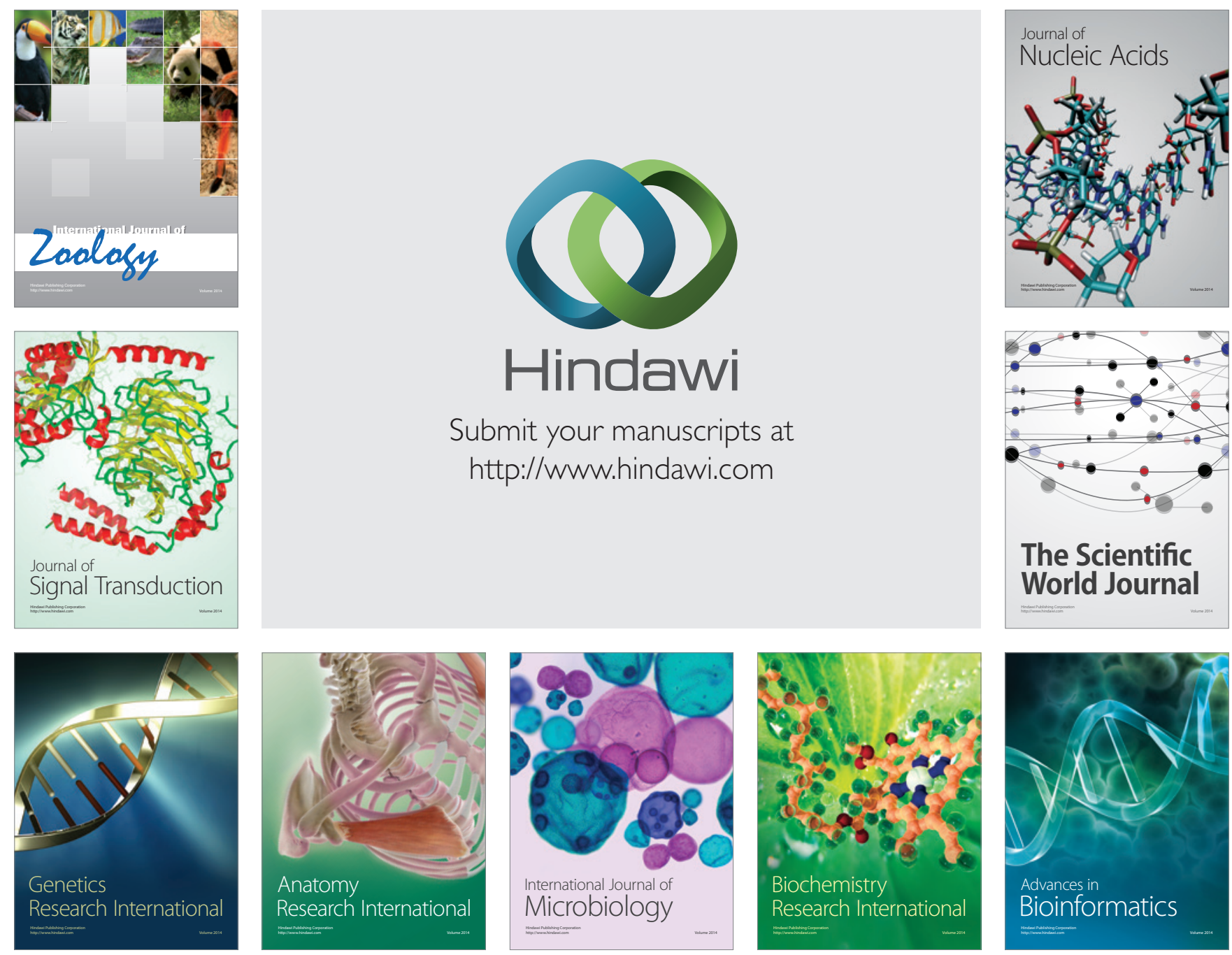

The Scientific World Journal
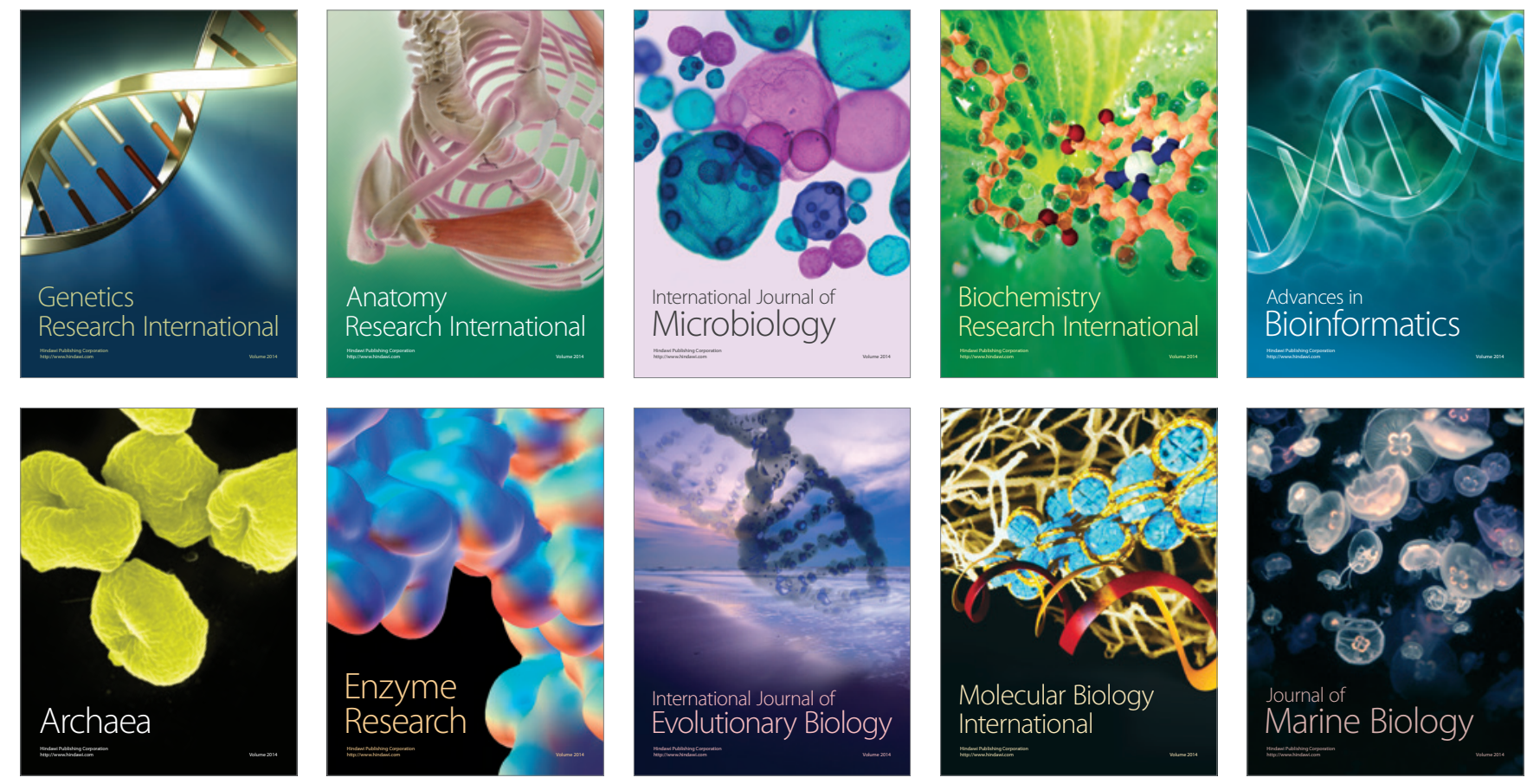